\title{
Evaluation of Schistocytosis in Patients Treated with Dialysis
}

\section{Journal of \\ Hematology \& Thrombosis}

Keywords: Schistocytes; Dialysis; Clinic al epidemiology

Abstract

Schistocytes are associated with micro angiopathic hemolytic processes. In this retrospective study; we evaluate the association of schistocytosis in the peripheral blood smear of patients before and after dialysis. We identified 7000 patients in our hospital system that underwent dialysis. The peripheral blood smears of 153 who were evaluable for the presence of schistocytosis prior to and after dialysis. Ninety seven patients had altemative reasons to have schistocytosis. Among the remaining 50 patients, $14 \%$ had schistocytes present prior to begin dialysis and $86 \%$ after one or more dialysis episodes. We conclude that the presence of schistocytes in the peripheral blood prior to dialysis is likely due to the underlying cause of renal impaiment or other co-morbidity. However, upon initiation of dialysis the proportion of patients with sc histocytosis is markedly inc rea sed and is likely due to the fragmentation of red cells during the dialysis procedure.

\section{Introduction}

A common reason for a hematology consultation in our medical center is the presence of schistocytes in the peripheral blood smear. In normal subjects schistocytes generally comprise less than 0.5 percent of the red cell population. Since schistocytes are often thought to be present when they are in fact artifactual, an experienced observer and appropriately prepared peripheral blood smear is a necessity. There is no clinical data whether dialysis therapy causes schistocytes due to mechanical injury or any other unknown pathological process. We have observed many patients with no other identifiable cause for schistocyte formation who are receiving dialysis. Schistocytes are fragmented red cells which may take a variety of shapes and are usually associated with mechanical disruption of the red cell membrane. The recognition of schistocytosis is important, since its existence may signal the presence of a life threatening condition. Schistocytes have been reported to occur in patients with vasculitis, disseminated intravascular coagulation (DIC), thrombotic thrombocytopenic purpura/hemolytic uremic syndrome (TTP/HUS), malignant hypertension, and in patients with intravascular grafts, devices, or stents placement $[1,2]$.

Although the occurrence of schistocytosis in patients undergoing dialysis has been previously described, the frequency and severity of this abnormality in this population is not known. Also whether red cell fragmentation is present due to the dialysis procedure or the underlying reason for dialysis remains uncertain [3-6].

It is therefore important to document the frequency of schistocytosis among patients undergoing dialysis and determine its clinical significance in this setting. This study evaluates the association of schistocytosis in patients both before and after dialysis.

\section{Methods}

\section{Data collection and statistical analysis}

We performed a retrospective case series study at our multi-

\begin{abstract}
Abuzar Moradi Tuchayi and Roy E. Smith*
Division of Hematology/Oncology, University of Pittsburgh Medical Center, Pittsburgh, PA, USA
\end{abstract}

*Address for Correspondence:

Roy E. Smith, Division of Hematology/Oncology, University of Pittsburgh Medical Center, Pittsburgh, PA, USA, E-mail: smithre@upmc.edu

Submission: 17 January, 2015

Accepted: 04 March, 2015

Published: 10 March, 2015

Reviewed \& Approved by: Dr. Kerry Hitos, Executive Director, Department of Surgery, University of Sydney, Australia

institutional center. This study has been approved by Institutional review board and ethics approval was obtained. We reviewed 7000 patients identified by the hospital Medical Archival Retrieval System (MARS) laboratory data system who underwent dialysis from January 2000 - December 2013. The MARS query included the physical examination, hospital course, laboratory data, and peripheral blood smear interpretation. Complete blood counts $(\mathrm{CBC})$ were determined with a Coulter DXH automated Beckman-Coulter blood cell counter using blood samples obtained in an EDTA containing vacutainer. The CBC results during each patient's course were reviewed. Values for each major element of the CBC are reported here only when there was no identifiable confounding event recorded in the medical record. For the purposes of this study, only the lowest platelet counts were collated. The prothrombin time (PT) and activated partial thromboplastin time (APTT) were performed using a Stago Star Evolution coagulation analyzer. Prothrombin times and APTTs were included exclusive of those obtained less than six hours after the dialysis procedure in order to eliminate confounding due to anticoagulant exposure during dialysis.

After selecting the patients whose peripheral blood smears were evaluated before and after dialysis, we collected the demographic information which included age, gender and race. We evaluated each patient for changes in the total white blood cell and granulocyte counts, the occurrence of thrombocytopenia or thrombocytosis, and changes in the prothrombin time and activated partial thromboplastin time. The electronic medical record for each patient was thoroughly reviewed for causes of red cell fragmentation. Patients with vasculitis, DIC, TTP/ HUS, malignant hypertension, and those with intravascular grafts, devices, or stents were excluded from analysis.

Blood cell morphology was assessed by certified laboratory hematological technicians examining each peripheral blood smear in its entirety and in the optimal location for assessing red morphology with a 100x oil immersion lens. A red cell was determined to be fragmented if it was a helmet cell, triangulocyte, pre-keratocyte (blister cell); all with lack of central pallor (see Figure 1) in accordance with the International Council on Standardization of Hematology guidelines [7]. 


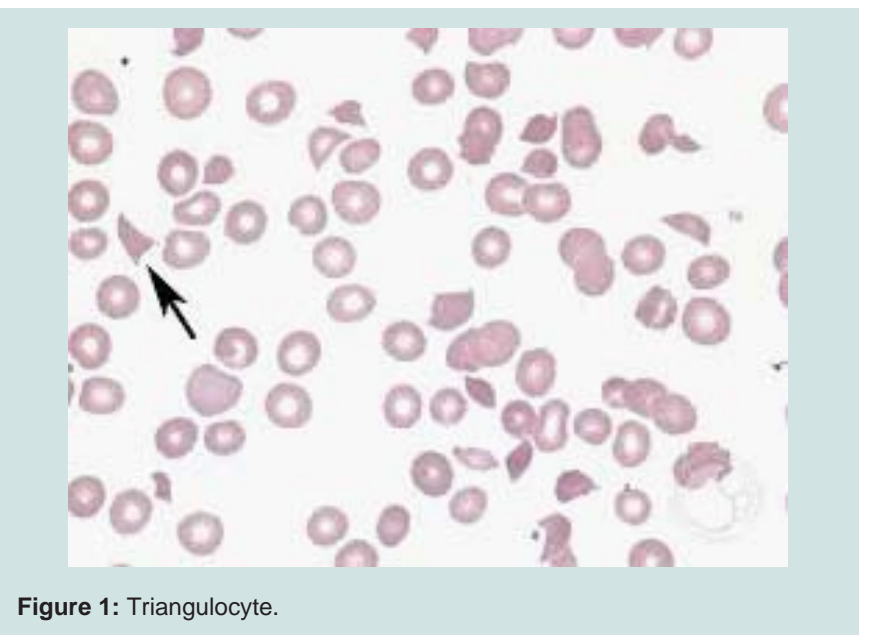

Red cells with questionable morphology were routinely examined by a laboratory supervisor and appropriately categorized. If there were further concerns regarding the interpretation of abnormal red cell morphology, the peripheral blood smear was reflexively reviewed by an attending hematopathologist. All peripheral blood smears of patients seen in consultation by the hematology consult service for our institution were reviewed by a hematology attending physician. The number of fragmented red cells were reported as a proportion of each one hundred red cells assessed and designated as being occasional ( $<1$ fragmented red cell $), 1+(1-3$ fragmented red cells), $2+$ (3-6 fragmented red cells), 3+ (6-12 fragmented red cells ), and 4+ ( $>12$ fragmented red cells ) according to American Society of Clinical Pathology guidelines [8].

All data were entered into Microsoft excel worksheet and statistical analysis performed using SPSS Version 22. The mean, minimum and maximum range was used to describe non parametric continuous variables such as age. All other values are expressed as percentages. McNemar test was used to assess relationships between groups. Two-sided P-value $<0.05$ was designated as significant. Relative risk of schistocytosis was calculated.

\section{Results}

One hundred fifty three patients were evaluated for the presence of schistocytes in their peripheral blood smear. Mean age was 60 (23-89). Seventy six patients (49\%) were males. Seventy seven patients (51\%) were females. Sixty nine patients (45\%) were African American. Eighty four patients (55\%) were Caucasian. One hundred forty seven patients (96\%) underwent hemodialysis. Five patients (3\%) underwent peritoneal dialysis. One patient $(0.6 \%)$ underwent hemodialysis and peritoneal dialysis.

The absolute granulocyte count (AGC) was normal in one hundred eleven patients (72.5\%). Of those patients who had the AGC fall outside of the normal range, forty patients (95\%) had an increased AGC and two patients (5\%) had a decreased AGC. Eleven patients (72\%) had thrombocytosis, 55 patients (35.9\%) had thrombocytopenia, and 87 patients $(56.9 \%)$ had normal platelet counts.
Twenty seven patients (21.3\%) had a prolonged APTT. Twenty three patients (18.1\%) had a prolonged PT, and twenty six patients (17\%) had both tests prolonged. No patient had either the APTT or PT less than the normal range for this institution. During the course of their dialysis twenty two patients had an episode of superficial or deep vein thrombosis. All were related to either line placement or shunt maintenance.

No patient had evidence of venous embolization. No patient had vasculitis, TTP, malignant hypertension, intravascular grafts, or evidence of obstructions within the extracorporeal circuit, kinking of blood tubing, excessive flow, improper cannula, or catheter dimensions. One patient $(0.65 \%)$ was diagnosed as having hemolytic uremic syndrome. Twenty six patients (17\%) had evidence of DIC. Seven patients (4.5\%) had arterial line placement. Thirty five patients (23\%) had indwelling catheters inserted. Forty seven patients (31\%) were using eithertacrolimus or cyclosporine.

After excluding patients with definite reasons for developing schistocytosis, we found fifty patients who developed schistocytosis without an alternative pathological mechanism. Twenty seven patients (54\%) developed Schistocytosis on the same day and after undergoing dialysis. Twenty eight patients (56\%) had AV fistula while twenty two patients (44\%) had dialysis catheter. No patient had blood transfusion.

The distribution of schistocytosis in patients before and after dialysis is shown in Figure 2.

The relative risk of schistocytosis was $(\mathrm{RR}=6.143$, CI 3.063$12.321 ; \mathrm{P}<0.0001$ ). Only $14 \%$ (seven) patients presented with schistocytes in the peripheral blood prior to dialysis: however, the occurrence of schistocytosis increased precipitously after dialysis was performed (86\%: 43 patients). No patient had prior to or after dialysis an ASCP schistocytosis score greater than (1+). The difference in the proportion of patients with schistocytosis pre and post dialysis was highly significant. No patient developed further evidence of thrombotic microangiopathy during the course of dialysis and illness.

\section{Discussion}

The presence of schistocytosis in patients with renal impairment or those receiving renal replacement therapy is often a dilemma for the consulting clinician. These patients are frequently complex with competing morbidities and multiple medications being administered.

In this series of patients, we excluded the more common designated causes of schistocytosis and queried our laboratory database to be certain that these mechanisms of schistocytosis did not apply. In most instances the presence of DIC and TTP- HUS were excluded simply by the course of the patient's illness.

Although all of these patients had renal impairment requiring dialysis, none had convincing evidence for the presence of a systemic thrombotic predisposition as typically seen in patients with TTP and some patients with HUS. Although mild thrombocytopenia was present in a small number of patients, it was often transient and did not worsen over time.

Most previously published studies only determined helmet cells to be schistocytes. In this study we considered all the different 


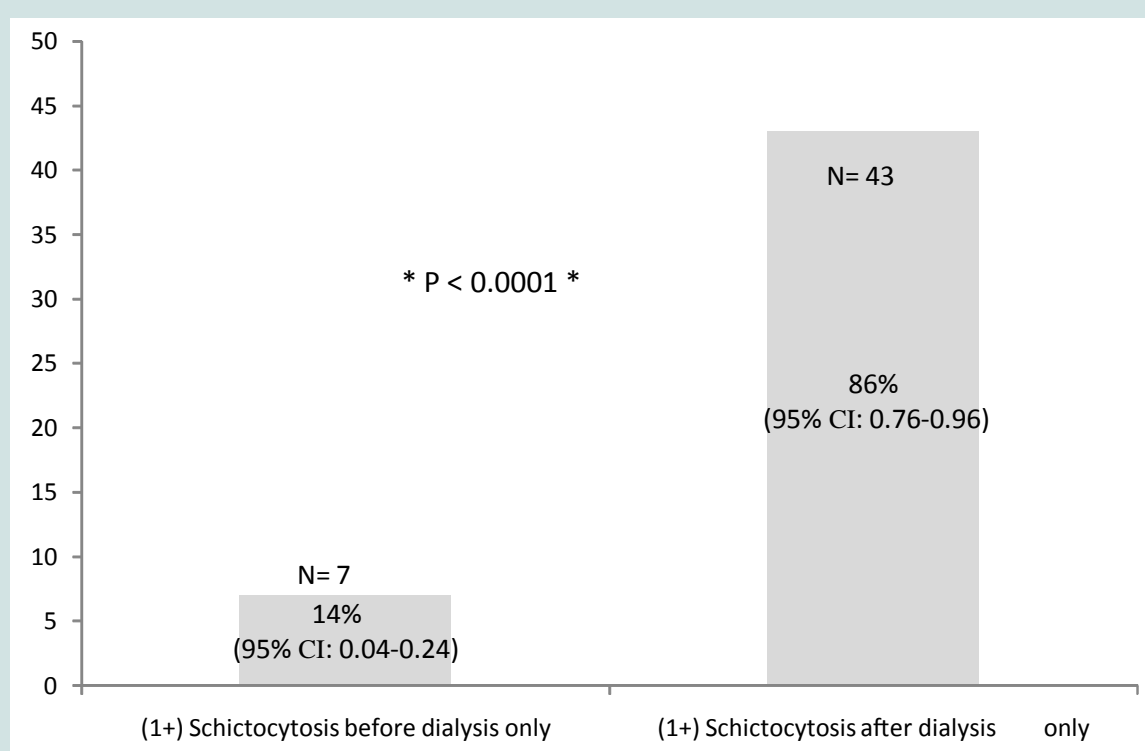

Figure 2: Distribution of schistocytosis before and after dialysis.

shapes of the schistocytes including helmet cell, triangulocytes, prekeratocytes (blister cells) and keratocytes (horncells).

After excluding the patients who had definite alternative reasons for schistocytosis, we found only $14 \%$ of patients had schistocytes detected in their peripheral blood smear prior to dialysis, and that the proportion of patients with detectable schistocytes increased markedly (86\%) after dialysis. Based upon extensive reviews of each patient's prolonged medical course, we were unable to identify alternative explanations for the presence of red cell fragmentation in this cohort of patients.

Significantly, those patients who developed schistocytosis after dialysis did so on the day the procedure was performed. The absence of obvious other causes of schistocytosis prior to dialysis strongly indicates that this baseline hematological abnormality is likely related to the patient's underlying renal impairment or some other unidentified co-morbidity. The temporal relationship between dialysis and the occurrence of schistocytes among this same population of patients indicates that their presence is related to an extra corporeal disruption of red cell membranes during the procedure.

Extracorporeal damage to red cells and subsequent mechanical hemolysis is an unavoidable side effect of hemodialysis. Acute hemolysis can also occur, mostly due to mechanical issues (e.g., obstructions within the extracorporeal circuit, kinking of blood tubing, excessive flow, improper cannula or catheter dimensions) [9], but also due to the administration of hypotonic or hypertonic dialysate solutions. These mechanisms do not appear to contribute to the schistocytosis seen in our patients.

This is the first detailed description of schistocytosis in patients before and after dialysis and in the absence of any other identifiable cause. The severity of the schistocytosis is mild and might be easily overlooked by the inexperience observer. On the other hand, in our experience the report of the presence of schistocytes in the peripheral blood smear of these patients often result in an unnecessary hematology consultation. We demonstrate that schistocytosis of mild severity is present in a relatively small proportion of patients with renal failure and that the large majority of patients undergoing dialysis develop schistocytosis as a result of the procedure. Therefore patients with greater than (1+) schistocytosis who undergo dialysis should be evaluated for other comorbidities. On the other hand, the post dialysis occurrence of schistocytosis of $1+$ or less severity is likely related to the procedure. The presence of this abnormality, in this setting, does not necessarily indicate the presence of comorbidity with dire consequences.

This study has several strengths and weaknesses. Among the strengths are the large number of patients with peripheral blood smears assessed for the presence of red cell morphological abnormalities over many years by competent individuals applying standardized methods and criterion which reduced the play of selection bias. Another strength is the exclusion of co-morbidities or drug-effects. Weaknesses include the retrospective design, the initial selection and exclusion of patients which was based upon data derived from the electronic medical record, and the number of patients which had to be excluded from this assessment due to lack of complete data availability.

This study demonstrates that $1+$ schistocytosis, as designated by the American College of Pathology criteria, is commonly seen in patients undergoing dialysis for renal impairment. The presence of greater than 1+ schistocytosis may indicate to the clinician that alternative diagnostic possibilities are present. We plan to use these findings to design a prospective study of the occurrence and severity of red cell morphological abnormalities among patients receiving dialysis according to the duration of treatment, types of dialysis equipment used, and flow rates.

\section{References}

1. Rosse W, Bunn HF (1994) Hemolytic anemias. In: Harrison's Principles of 
Citation: Tuchayi AM, Smith RE. Evaluation of Schistocytosis in Patients Treated with Dialysis. J Hematol Thromb 2015;1(1): 4.

ISSN: $2380-6842$

Internal medicine, (13th Ed), eds. Isselbacher KJ, Braunwald E, Wilson JD, Martin JB, Fauci AS, et al. McGraw Hill, USA $1743-1757$

2. Grimm J, Brunn H, Heller M, Mueller-Huelsbeck S (2003) Hemolytic effect of deformed intra-arterial stents and stent grafts in vitro. Eur Radiol 13:1333 1338.

3. Fishbane S, Paganini E (2006) Hematologic abnormalities. In: Handbook of Dialysis. Daugirdas JT, Blake PG, Ing TS. Lippincott Williams \& Wilkins, USA 536-537.

4. Rodriguez Perez JC, et al. (1986) Hemolysis during Hemodialysis. Dial Transplant 15: 334-335

5. Nand S, Bansal VK, Kozeny G, Vertuno L, Remlinger KA, et al. (1985) Red cell fragmentation syndrome with the use of subclavian hemodialysis catheters. Arch Intern Med 145: 1421-1423.

6. Vercaemst L (2008) Hemolysis in cardiac surgery patients undergoing cardiopulmonary bypass: a review in search of a treatment algorithm. J Extra Corpor Technol 40: 257-267.

7. Zini G, Onofrio G, Briggs C, Erber W, Jou JM, et al. (2012) ICSH recommendations for identification, diagnostic value, and quantitation of schistocytes. Int J Lab Hematol 34: 107-116.

8. Brown BA, Hunter RC, O'Hare A, Erim G (1993) Hematology: principles and procedures. Lea \& Febiger, Philadelphia.

9. Polaschegg HD (2009) Red blood cell damage from extracorporeal circulation in hemodialysis. Semin Dial 22: 524-531. 